\title{
Cell adhesion molecules in neural development and disease
}

This Special Issue focuses on the significance of the expression of neural cell adhesion molecules in development and disease. These molecules may belong to different gene families and may be involved in distinct developmental events with Cadherins, Integrins, the Semaphorins and the Immunoglobulin Superfamily representing the main groups (Hayashi and Takeichi, 2015; Gardiner, 2011; Battistini and Tamagnone, 2016; Irintchev and Schachner, 2012). This SI mostly focuses on components of the last family (IGSF). Early studies demonstrated that expression of these molecules was critical both during development and in the adult nervous tissue and was responsible for the ability of cultured neural cells to undergo an adhesive behaviour. However, the advent of gene targeting technologies, particularly in mice, made it evident that many of these so-called adhesion molecules were rather endowed with complex developmental functions and that their adhesive properties should perhaps be regarded as an epiphenomenon of their functional make up. This led to the early definition of these cell surface glycoproteins as ‘morphoregulatory' molecules (Edelman, 1992), reflecting that such proteins are specifically involved in the complex mechanisms modulating the shape of nervous tissues. Encompassed in this concept was the idea that rather than simply acting as glue, such molecules transmit transmembrane signals involved in developmental control and that, as such, they should be regarded as signalling molecules, whose ultimate role was to regulate neural morphology at cellular and tissue levels. A corollary of this assumption is that precise regulation of the genes encoding these molecules is a critical element of their developmental function and, therefore, that alteration in their expression profiles could result in neurological disorders. The different contributions of this Special Issue focus on different aspects of this general topic.

The review article from Takeshi Sakurai (Sakurai, 2016) deals in general with the significance of changes of CAM expression and function in neuropsychiatric disorders and provides a framework for thinking about the different levels at which alterations to circuitry may occur. Although mutations in CAMs are known to be causal in neurological disease, most notably in the L1CAM-associated MASA/CRASH syndromes, more typically rare genetic or copy number variants have been found associated, among others, with intellectual disability (ID), autism spectrum disorder (ASD), schizophrenia (SZ) and peripheral neuropathies. Focussing particularly on the fact that CAMs are often incorporated in multimeric complexes, in which changes in individual components may lead only to subtle changes in neuronal circuitry, the author contends that CAM genes are important 'risk genes' in neuropsychiatric disorders and suggests five ways in which such subtle changes may make the organism vulnerable to neurological disease. Most obviously this may result from the known importance of CAMs to the function of mature circuits, and he highlights roles in the trafficking of synaptic components which may affect homeostatic plasticity. However, this may also result from circuitry modification during development, arising from effects on early differentiation (neuronal specification/axonal growth/fasciculation), but also from later-activated mechanisms, such as target recognition, synapse formation and myelination, and involving diverse adhesion families, including Cadherins, Ig superfamily and Integrins, as well as members of the Semaphorin family.

The assumption that changes in the expression of the morphoregulatory glycoproteins encoding genes may contribute to neurological disorders is consistent with the general idea that the activation profile of the underlying genes is a critical aspect of their function and that changes in their profile may be causative in specific pathologies of the nervous system.

The review from Alexander Dityatev and Hristo Varbanov (Varbanov and Dityatev, 2016) concerns one of the most widely characterized adhesion molecules of the nervous tissue: the Neural Cell Adhesion Molecule (NCAM). Rather than focusing on its adhesive properties, the manuscript summarizes the relationship of NCAM with the functions of neural circuits, in particular with synaptic plasticity, through its activation of the $N$-methyl-D-aspartate-receptors (NMDARs), revealing distinct roles for the NCAM polypeptide and its polysialic acid modification in different circuits. A role for the polysialylated form of the NCAM (PSA-NCAM) acting on extrasynaptic NMDAR is also discussed. This interaction is thought to inhibit the activation of extrasynaptic NMDAR which otherwise would inhibit LTP and activate cell death. Polysialylated NCAM interactions with the extrasynaptic form of the NMDAR thus results in positive effects on long term potentiation and counteracts neurodegeneration and in the hippocampus and medial prefrontal cortex promotes neurogenesis, and enhances synaptic plasticity and learning. Interestingly, much of this suppression appears to be mediated by soluble PSA-NCAM and the review explores the mechanisms leading to PSA-NCAM shedding from GABAergic interneurons, which indicates that this may involve Ephrin family components and activation of ADAM proteases.

The authors also explore the association of alterations of PSA-NCAM expression with neuropsychiatric disorders, notably with schizophrenia, bipolar disorder, depression anxiety and Alzhemier's disease, and examine the potential of PSA as a therapeutic agent in such diseases.

Andreas Faissner and colleagues (Faissner et al., 2016) focus on the interactions of adhesion molecules with extracellular matrix components which, together with other ECM-associated proteins, comprise the set of proteins encoded by the genes of the so-called matrisome. The authors are particularly concerned with the elucidation of the ECM interactome associated with the neural stem cell niche, most specifically with Tenascin C (Tnc), a component of the tenascin family, which is presented as a model of ECM components specific to the nervous system. Its role in the modulation of proliferation and 
differentiation events of neural precursor cells, with particular reference to stem cells and their role in neural regeneration is discussed. Aside from being a ligand for Integrins and Contactins, Tnc is also known to bind cytokines and morphogens (TGFbeta1, wnt3a, PDGF, FGF2, NT3) and stimulate the EGF receptor via its EGF repeats. Perhaps not surprisingly then, it is highly expressed in malignant glial tumors and is upregulated in CNS lesions, all consistent with a critical role in regulating neural precursor behaviour.

The remaining reviews of this Special Issue take a tour through three different families of CAMs of the immunoglobulin supergene family that each have striking expression in different aspects of neural development, from expression on early progenitors through clear roles in synaptogenesis and myelination, though the overall lesson is that trying to tie a specific molecule to a single stage of development is futile and instead nature efficiently reuses its tools again and again.

Fritz Rathjen and colleagues (Matthäus et al., 2016) deal with the Cosackievirus and Adenovirus Receptor (CAR)-like cell adhesion molecules family, a less well-studied subfamily of the Ig superfamily, comprising CAR itself, CLMP (CAR-like membrane protein) and BT-IgSF (brain and testis specific immunoglobulin superfamily). Although widely expressed in a variety of tissues, these exhibit a striking profile in nervous tissues, CAR and CLMP being highly expressed in development but replaced by BT-IgSF, which shows the reciprocal pattern as maturation proceeds. CAR proteins are transmembrane proteins built by the association of a $\mathrm{N}$-terminal $\mathrm{V}$ type domain with a C-terminal C2 domain, both involved in homotypic cell adhesion. These proteins are concentrated at specific sites of cell communications, the gap and tight junctions. Although the only disorders associated with this family in humans so far relate to the association of gastrointestinal disease with mutations in CLMP mutation, CAR knockout mice exhibit behavioural changes that may be associated with impaired hippocampal neurogenesis, while BT-IgSF knockouts are disrupted in aspects of AMPAR-mediated synaptic transmission in the hippocampus. Since CAR levels appear reduced in the inflamed hippocampus of patients with late onset Alzheimer's disease, this may indicate a role for CAR proteins in neurodegenerative disorders.

Esther Stoeckli (Frei and Stoeckli, 2016) turns to the so-called synaptic cell adhesion molecules (Syn CAMs). These are Immunoglobulin superfamily components which classically have the property of being able to induce synapse formation in non-neuronal cells and as such they may be considered as true synaptic cell adhesion molecules. However, as Stoeckli discusses, they also mediate axo-glial contacts formation, indicating their involvement in myelination and, in addition, have recently been shown to have roles in axon guidance in both the central and peripheral nervous tissue, suggesting that SynCAMs may in fact, like many of the families discussed in this Special Issue, also be 'Jacks of all trades' in neurodevelopment. Correspondingly, mutations in SynCAM genes are also identified as causal or risk factors in neurodevelopmental disorders, notably for intellectual disability and autism spectrum disorders, and knockout mice exhibit social and emotional behaviour deficits.

The Contactin (Cntn) subset of the Immunoglobulin supergene family (IgC2/FNIII molecules) (Gennarini et al., 2016) includes molecules which share structural properties (the association of Immunoglobulin type C2 with Fibronectin type III domains), as well as a general role in cell contact formation and in axonal growth control. This subset includes 6 highly related components (Contactin 1 to Contactin 6) all of them associated with the cell membrane via a Glycosyl Phosphatidyl Inositol (GPI)-containing lipid tail. The highest similarity is shared by Contactin 1 and Contactin 2 ( $~ 50 \%$ at the protein level). These are cell surface components, which bind heterophilically to multiple partners including members of the related L1CAM and of the Neurexin family (Contactin-associated proteins CNTNAPs or CASPRs).

Besides the organization of the neuronal membrane, these molecules also contribute to axonal growth and pathfinding control and promote axonal interactions with myelinating cells at the paranodal and juxtaparanodal regions, leading to differential ionic channels (both $\mathrm{Na}^{+}$and $\mathrm{K}^{+}$) distribution, an event of critical relevance in the generation of the peak-shaped action potential.

The Contactins also appear to have a role in degenerative and inflammatory disorders. Contactin 2 is suggested to be involved in Alzheimer's disease and is a known ligand of the Alzheimer Precursor Protein (APP), binding to which promotes release of Alzheimer Intracellular domain (AICD) and negatively modulates neurogenesis. Contactin- 1 overexpression also has effects on neurogenesis, but appears to do so through activation of Notch signalling via the Hes pathway. Activation of Notch signalling may also be consistent with its ability to modulate neuroinflammation events, and with the possibility that Contactin 1-dependent interactions may participate in the pathogenesis of neuroinflammatory disorders, including multiple sclerosis.

The contribution from Daniela Puzzo (Gulisano et al., 2016) and colleagues extends the analysis of Contactin 1 function by exploring its role in hippocampal synaptic plasticity and in memory in both adult and aged mice, achieved by studying long-term potentiation as well as spatial and recognition memory. They investigate the molecular pathways underlying these functions and demonstrate the involvement in such events of the CREB transcription factor phosphorylation. With potential significance for neurological disorders, the molecule was also found to influence neuronal apoptosis, including the production of amyloidbeta peptide $(A \beta)$, and therefore, as for Contactin 2, also Contactin 1 was proposed to be one of the pathogenetic hallmarks of Alzheimer's disease (AD).

Finally, Peter Burbach and colleagues (Oguro-Ando et al., 2016) review recent genetic studies that implicate Contactins 4,5 and 6 as candidate genes in neuropsychiatric disorders, particularly autism spectrum disorders, intellectual disability, schizophrenia, attention deficit/hyperactivity disorders, bipolar disorders, alcohol use disorders and Anorexia Nervosa. This is consistent with the studies on the developmental function of these proteins, which demonstrate roles in cell survival, neurite growth and pathfinding, and on subsequent circuit formation. These studies also indicate the existence of a differential code for Contactin expression within the different neural regions including the retina, the cerebral cortex and the thalamus, which in turn may differentially affect the developmental organization of these structures.

Overall, it may be supposed that the modulation of the Contactin code is of central relevance in neural developmental control and that, in turn this may result in the definition of the binding specificity of the neuronal surface. However, besides trans-interactions with distinct ligands, this certainly involves cis-association of these GPI-anchored molecules with transmembrane partners, which in turn allows them to interact with the submembrane compartment. Therefore these different mechanisms based on both cis-association and trans-interactions of the so called cell adhesion molecules both contribute to build the complex molecular machine, which, while modulating the nervous tissue differentiation during physiological development may also mediate the neural damage in specific circumstances.

\section{Where now?}

Add to the molecular complexity detailed above, and in the Special Issue reviews, the overlying complexity and robustness of the circuits being built, and it becomes clear that simply knocking out or overexpressing genes and then looking either at the static anatomy of the resulting circuits, or the behaviours of the lab animals is only going to get us so far, particularly when environmental insults are major contributors to the triggering of neurological disease, as seems to be the case. What clearly is needed is the ability to observe the activity of circuits in the behaving living animal while protein functions are altered in real time. Despite advances in powerful genetics and in vivo imaging (e.g. http://www.nature.com/nmeth/journal/v12/n2/abs/nmeth.3217. html), achieving this goal in the mouse still appears to remain a long way off. Thus, while the mouse undoubtedly will remain the workhorse in this area, not least because of the wealth of experience with this 
model and its relevance to humans, it may nonetheless be time for those studying the roles of CAMs in the formation and function of neural circuits to consider the usefulness of simpler more tractable systems, such as the zebrafish, where recent advances in genetics and imaging are now making this goal look attainable (e.g. https://www.ncbi.nlm. nih.gov/pmc/articles/PMC5282529/).

\section{Acknowledgements}

AJWF supported by funding from Yorkshire Cancer Research and Brain Tumour Research and Support across Yorkshire.

\section{References}

Battistini, C., Tamagnone, L., 2016. Transmembrane semaphorins, forward and reverse signaling: have a look both ways. Cell. Mol. Life Sci. 73, 1609-1622.

Edelman, G.M., 1992. Morphoregulation. Dev. Dyn. 193, 2-10.

Faissner, A., Roll, L., Theocharidis, U., 2016. Tenascin-C in the matrisome of neural stem and progenitor cells. Mol. Cell. Neurosci. http://dx.doi.org/10.1016/j.mcn.2016.11. 003.

Frei, J.A., Stoeckli, E.T., 2016. SynCAMs - from axon guidance to neurodevelopmental disorders. Mol. Cell. Neurosci. http://dx.doi.org/10.1016/j.mcn.2016.08.012.

Gardiner, N.J., 2011. Integrins and the extracellular matrix: key mediators of development and regeneration of the sensory nervous system. Dev. Neurobiol. 71, 1054-1072.

Gennarini, G. Bizzoca, A., Picocci, S., Puzzo, D., Corsi, P., Furley, A.J. 2016. The role of GPIanchored axonal glycoproteins in neural development and neurological disorders. Mol. Cell. Neurosci. http://dx.doi.org/10.1016/j.mcn.2016.11.006.

Gulisano, W., Bizzoca, A., Gennarini, G., Palmeri, A., Puzzo, D., 2016. Role of the adhesion molecule F3/Contactin in synaptic plasticity and memory. Mol. Cell. Neurosci. http://dx.doi.org/10.1016/j.mcn.2016.12.003.
Hayashi, S., Takeichi, M., 2015. Emerging roles of protocadherins: from self-avoidance to enhancement of motility. J. Cell Sci. 128, 1455-1464.

Irintchev, A., Schachner, M., 2012. Injured and regenerating nervous system: immunoglobulin superfamily members as key players. Neuroscientist 18, 452-466.

Matthäus, C., Langhorst, H., Schütz, L., Jüttner, R., Rathjen, F.G., 2016. Cell-cell communication mediated by the CAR subgroup of immunoglobulin cell adhesion molecules in health and disease. Mol. Cell. Neurosci. http://dx.doi.org/10.1016/j.mcn.2016.11.009.

Oguro-Ando, A Zuko, A Kleijer, KT. Burbach, JP. 2016. A current view on Contactin-4, -5 , and -6 : implications in neurodevelopmental disorders. Mol. Cell. Neurosci. http:// dx.doi.org/10.1016/j.mcn.2016.12.004.

Sakurai, T., 2016. The role of cell adhesion molecules in brain wiring and neuropsychiatric disorders. Mol. Cell. Neurosci. http://dx.doi.org/10.1016/j.mcn.2016.08.005.

Varbanov, H., Dityatev, A., 2016. Regulation of extrasynaptic signaling by polysialylated NCAM: impact for synaptic plasticity and cognitive functions. Mol. Cell. Neurosci. http://dx.doi.org/10.1016/j.mcn.2016.11.005.

Gianfranco Gennarini

Department of Basic Medical Sciences, Neurosciences and Sensory Organs. Medical School. Policlinico, Piazza Giulio Cesare 11, 70124 Bari, Italy

Corresponding author.

E-mail address: gianfranco.gennarini@uniba.it.

Andrew Furley

Department of Biomedical Science, University of Sheffield, Western Bank, Sheffield S10 2NT, UK 